\title{
Análise de Ferramentas de Suporte às Tarefas do Professor-Tutor em um Ambiente Virtual de Ensino e Aprendizagem
}

\author{
Fábio G. Andrade ${ }^{1}$, Maurício C. Rosito ${ }^{1}$, Júlia M. C. da Silva ${ }^{1}$ \\ ${ }^{1}$ Curso Superior de Tecnologia em Análise e Desenvolvimento de Sistemas \\ Instituto Federal de Educação, Ciência e Tecnologia do Rio Grande do Sul (IFRS) \\ Av. Osvaldo Aranha, 540 - 95.700-000 - Bento Gonçalves - RS - Brazil \\ fagoand@hotmail.com, \{mauricio.rosito,julia.silva\}@bento.ifrs.edu.br
}

\begin{abstract}
Virtual Learning Environments (VLEs) consist of systems for mediating educational activities, which can be used in classroom courses, semi-distance and distance courses. However, difficulties experienced by teachers indicate an underutilization of such tools, whereas related research tend to focus on the needs of students. This work sought to investigate whether the difficulties in using VLEs are related to the lack of tools that provide some sort of support for teachers and tutors. Based on a review of related work and an electronic questionnaire administered to teachers users of VLE, seven plugins for the Moodle environment were developed, in order to verify if the development of new tools could promote a better usage of the virtual environment by the teachers-tutors.
\end{abstract}

Resumo. Os Ambientes Virtuais de Ensino e Aprendizagem (AVEAs) são sistemas para mediação de atividades didáticas em cursos presenciais, semipresenciais e a distância. Entretanto, dificuldades vivenciadas por docentes apontam para um subaproveitamento das ferramentas, enquanto pesquisas relacionadas tendem a focar nas necessidades do aluno. O presente trabalho buscou investigar se as dificuldades no uso de AVEAs estão relacionadas à falta de ferramentas que ofereçam suporte a professorestutores. A partir de uma revisão de trabalhos correlatos e um questionário aplicado a professores usuários de AVEA, foram desenvolvidas 7 ferramentas para o ambiente Moodle, a fim de verificar se o desenvolvimento de novas ferramentas pode promover um melhor uso do AVEA por professores-tutores.

\section{Contextualização}

O uso de recursos tecnológicos aplicado ao contexto didático é um tema recorrente na área da Educação. Em uma sociedade onde a produção e disseminação de conhecimentos se dá de forma ampla e veloz, o professor é desafiado a assumir uma nova postura diante da tecnologia [Mercado 2002].

Os Ambientes Virtuais de Ensino e Aprendizagem (AVEAs) consistem em sistemas informatizados voltados a educadores e educandos. Estes permitem a mediação de atividades didáticas mesmo quando as partes envolvidas não se encontram fisicamente no mesmo local. Por esta razão, são frequentemente utilizados em cursos de 
Educação a Distância (EaD) ou ainda como complemento em cursos presenciais e semipresenciais [Almeida 2003].

Mas ao mesmo tempo em que ferramentas digitais ampliam seu espaço na educação, ainda são comuns os relatos de professores que não conseguem incorporar tais recursos à prática docente. De modo geral, observa-se que os estudos de novas tecnologias aplicadas à educação costumam focar no ponto de vista do aluno [Kinshuk et al., 2001]. O professor-tutor, embora igualmente importante no processo ensinoaprendizagem, tende a ser subvalorizado, haja vista o número reduzido de pesquisas relacionadas à sua interação com tais ferramentas [Yacef 2002; Andrade 2016].

\section{Caracterização do Problema de Pesquisa}

As dificuldades relatadas por docentes no uso de ambientes virtuais - tais como a complexidade e tempo necessário para a realização de tarefas - acabam por limitar sua interação com os alunos e o potencial tecnológico do AVEA. Desta forma, a presente pesquisa se propôs a investigar se:

- Q1: As dificuldades no uso de AVEA estão relacionadas à falta de ferramentas que melhor suportem ao professor-tutor?

- Q2: A partir do desenvolvimento de novas ferramentas, é possível promover um melhor uso do ambiente virtual por professores-tutores?

Para responder a Q1, definiu-se a seguinte hipótese principal: "As dificuldades enfrentadas por professores e tutores no uso de AVEAs derivam da falta de ferramentas que ofereçam maior suporte à prática docente". A verificação desta hipótese foi realizada por meio de uma revisão sistemática de artigos e trabalhos relacionados, bem como de uma análise qualitativa e quantitativa de dados obtidos junto a professores usuários de AVEA. Tais informações possibilitaram a especificação e desenvolvimento de sete plugins para o Moodle (2015), ambiente virtual utilizado no IFRS - Campus Bento Gonçalves.

Com relação à Q2, foram definidas seis hipóteses secundárias com base no Modelo Afetivo de Tutores EaD [Cunha et al. 2008]. Assim, verificou-se se o uso das ferramentas propostas aumenta a pontualidade (H1), comprometimento (H2), comunicabilidade (H3), sociabilidade (H4), iniciativa (H5) e meticulosidade (H6) do professor-tutor nos cursos oferecidos em AVEA [Andrade 2016].

\section{Metodologia de Pesquisa}

O presente trabalho se classifica como uma pesquisa aplicada, visto que busca "gerar conhecimentos para aplicação prática, dirigidos à solução de problemas específicos" [Silveira and Córdova 2009]. O processo de coleta de dados se deu em três perspectivas distintas: consulta a produções bibliográficas relacionadas, questionários voltados a professores usuários de ambientes virtuais, e a análise de registros em AVEAs.

Durante a revisão sistemática, buscou-se identificar trabalhos correlatos e reconhecer as principais pesquisas relacionadas ao tema. A análise de artigos científicos e extensões para um ambiente virtual foi realizada em conjunto com os pesquisadores do Laboratório de Aprendizagem e Desenvolvimento de Software (LADS), do IFRS Campus Bento Gonçalves. 
Na sequência, um questionário eletrônico foi utilizado como instrumento para obtenção de dados. A amostra intencional foi composta por 355 professores e pesquisadores de diferentes instituições de ensino, caracterizando uma pesquisa com survey [Silveira and Córdova 2009].

Sete plugins para o ambiente Moodle foram especificados e posteriormente desenvolvidos pela equipe do LADS, empregando técnicas de programação, conhecimentos de banco de dados, interação humano-computador e inteligência artificial. O desenvolvimento foi seguido por uma pesquisa experimental [Silveira and Córdova 2009], enquanto a análise das potencialidades oferecidas pelas novas ferramentas se deu por meio de um estudo de caso no IFRS - Campus Bento Gonçalves.

A etapa seguinte consistiu em uma avaliação de como os professores utilizam AVEAs. Para isto, adotou-se a abordagem explicativa do tipo ex-post-facto. Este tipo de pesquisa investiga a relação de causa e efeito de um fenômeno após a sua ocorrência [Silveira and Córdova 2009]. Os dados analisados nesta etapa compreenderam registros de cursos (logs) em dois semestres distintos, todos no ambiente Moodle.

Finalmente, com base nas dimensões afetivas do professor, realizou-se a comparação entre o uso de AVEA antes e após o desenvolvimento dos plugins, através do teste estatístico Teste $t$ [Anderson et al. 2003]. Desta forma, tornou-se possível verificar as hipóteses principal e secundárias, ampliando-se a compreensão geral da perspectiva docente sobre o uso de AVEAs [Andrade 2016].

\section{Desenvolvimento da Pesquisa}

A primeira etapa do trabalho foi realizada entre os meses de março e junho de 2015 e consistiu exclusivamente na coleta de dados. Em uma revisão sistemática [Invernizzi et al. 2015], foram analisados 346 artigos publicados no evento MoodleMoot Brasil e 568 plugins disponíveis para o ambiente Moodle.

Desta amostra, constatou-se que apenas 15 artigos descrevem pesquisas que visam oferecer funcionalidades ao docente. Quanto aos plugins, foram observadas 107 alternativas com objetivo de auxiliar o professor em suas tarefas. Em ambos as categorias, os números obtidos foram considerados pouco expressivos quando comparados ao restante do conjunto.

Após investigados os recursos disponíveis para o professor-tutor usuário de AVEA, percebeu-se também a necessidade de verificar a opinião deste que é o públicoalvo das ferramentas a serem desenvolvidas. Para isto, foi elaborado um questionário eletrônico voltado a professores e pesquisadores usuários de AVEAs [Andrade 2016].

Em um intervalo de 7 dias no mês de abril de 2015, foram enviados 355 questionários a profissionais de diferentes instituições de ensino e pesquisa. Ao todo, 78 respondentes contribuíram com a pesquisa, caracterizando uma taxa de resposta de $22 \%$. A análise das respostas ao questionário auxiliou na identificação da visão do docente com relação aos ambientes virtuais que utilizam, destacando-se suas dificuldades e sugestões de melhorias.

Cerca de $90 \%$ dos entrevistados afirmou enfrentar algum tipo de problema no uso de ambientes virtuais. Dentre as dificuldades técnicas apontadas, destacaram-se a baixa usabilidade, bugs em módulos/ferramentas, acompanhamento de discussões 
(fórum) e o gerenciamento de múltiplos cursos. Outros fatores mencionados em grande parte das respostas foram: falta de tempo (para preparação de materiais e atendimento aos alunos), conhecimento digital limitado, resistência ou falta de estímulo para utilizar o ambiente e falta de controle sobre o ambiente em que as atividades são realizadas [Andrade 2016].

\section{Ferramentas Desenvolvidas}

A análise das respostas ao questionário aplicado a professores e pesquisadores indicou fatores críticos para professores-tutores usuários de AVEA, tais como o gerenciamento de múltiplos cursos e atividades. Segundo estes, controlar quais trabalhos foram enviados, quais tarefas precisam ser corrigidas e quais aspectos necessitam de sua atenção são atividades que exigem tempo e dedicação consideráveis por parte do professor-tutor, especialmente quando se referem a turmas numerosas ou a um grande número de cursos [Andrade 2016].

Assim, optou-se principalmente pelo desenvolvimento de ferramentas que pudessem auxiliar na organização de compromissos referentes a uma ou mais disciplinas no Moodle. Ao todo, foram desenvolvidos sete plugins para o ambiente Moodle, sendo estes um plugin de lembretes de tarefas (versões web e mobile), resumo semanal e diário de atividades e relatórios para administração de cursos.

\subsection{Plugin de Lembretes}

A primeira ferramenta desenvolvida apresenta um resumo centralizado de tarefas que necessitem da atenção do professor-tutor no ambiente virtual. As informações são exibidas logo na página inicial do Moodle e incluem: tarefas realizadas nos últimos 30 dias, trabalhos não avaliados, postagens não visualizadas em fóruns, dentre outras.

Todas as atividades listadas incluem nome, prazo (data e hora) e curso ao qual pertencem, além do número de itens já verificados e a quantidade que ainda precisa ser corrigida. O layout da ferramenta teve como base o plugin Archaius [Munera 2015], tema personalizado para o Moodle disponível no site do ambiente [Andrade 2016].

\subsection{Aplicativo para Dispositivos Móveis}

De maneira semelhante ao plugin de lembretes, o aplicativo IFRS-BG Moodle visa facilitar o gerenciamento de cursos no ambiente virtual Moodle por meio da centralização e síntese de informações. Do ponto de vista do professor, este pode ser um aliado na administração de tarefas, visto que reúne eventos relevantes de todos os cursos aos quais o usuário está associado [Andrade et al. 2015].

Após autenticar-se por meio de login/e-mail e senha, o usuário é redirecionado para a tela de lembretes, onde poderá visualizar notificações de próximas atividades, seguidas de registros dos últimos 30 dias. Caso haja tarefas a serem avaliadas, novas postagens em fóruns ou qualquer outro evento significativo desde o último acesso, estes serão exibidos em uma seção própria, discriminando-se o número de entradas novas e o total já verificado.

Além dos professores, alunos e seus responsáveis também podem se beneficiar do aplicativo, já que as notificações oferecidas permitem um acompanhamento direto da 
V Congresso Brasileiro de Informática na Educação (CBIE 2016)

Anais dos Workshops do V Congresso Brasileiro de Informática na Educação (CBIE 2016)

rotina escolar. Os usuários serão alertados quanto a provas, tarefas pendentes e demais atividades dos cursos a eles relacionados [Andrade et al. 2015].

\subsection{Relatórios de Atividades}

Os relatórios de atividades podem ser descritos como logs do AVEA para professorestutores, no sentido de que permitem visualizar as últimas interações registradas pelos usuários no ambiente virtual. O primeiro destes relatórios é chamado "Resumo da Semana" e relaciona as ações dos últimos 7 dias por curso, nome da tarefa/atividade, usuário e data/hora da ação.

Partindo do mesmo princípio, o relatório Resumo do Dia restringe o conteúdo exibido às ações das últimas $24 \mathrm{~h}$. Seu diferencial, no entanto, está no fato de que ele não pode ser visualizado dentro do AVEA, mas sim enviado automaticamente aos usuários por e-mail. Desta forma, após receber a lista de ações do dia, caberá ao professor-tutor decidir se deseja ou não acessar o Moodle [Andrade 2016].

\subsection{Relatórios Adicionais}

A criação de relatórios de acompanhamento esteve presente na maior parte das respostas ao questionário aplicado. Assim, além das ferramentas previamente descritas, outros três relatórios foram desenvolvidos para atender a necessidades específicas do processo ensino-aprendizagem em AVEAs. São eles: Relatório do Tutor, Relatório do Aluno e Relatório Perfil Afetivo do Tutor.

O Relatório do Tutor propõe um mecanismo para medir a atuação de professores-tutores ao longo de um curso. Tarefas corrigidas no ambiente virtual são contabilizadas e exibidas para cada professor-tutor do curso selecionado. As informações podem auxiliar no processo de gestão educacional, assim como na alocação de alunos por professor-tutor ou mesmo na autoavaliação destes profissionais.

O Relatório do Aluno realiza um processo semelhante, embora seu foco esteja no desempenho do aluno. Através dele, é possível verificar a participação dos estudantes em um determinado curso, observando-se os recursos visualizados, vídeos assistidos e uma série de outras interações realizadas por eles no AVEA.

Por fim, o relatório Perfil Afetivo do Tutor avalia professores-tutores de acordo com as seis dimensões afetivas do Modelo Afetivo de Tutores EaD [Cunha et al. 2008]: pontualidade, comprometimento, comunicabilidade, sociabilidade, iniciativa e meticulosidade. Para cada dimensão afetiva, uma escala de valores previamente definidos foi utilizada, de modo a classificar a média do professor como Muito bom, Bom, Regular, Ruim ou Muito Ruim [Andrade et al. 2016].

\section{Análise de Uso das Ferramentas}

Com exceção do Relatório Perfil Afetivo do Tutor, todas as ferramentas desenvolvidas foram disponibilizadas no Moodle do IFRS - Campus Bento Gonçalves a partir de setembro de 2015. O impacto destas soluções foi medido de duas maneiras, descritas a seguir. 
V Congresso Brasileiro de Informática na Educação (CBIE 2016)

Anais dos Workshops do V Congresso Brasileiro de Informática na Educação (CBIE 2016)

\subsection{Questionário de Aceitação}

O Questionário para docentes sobre as novas funcionalidades do Moodle IFRS-BG foi aplicado exclusivamente a 40 professores e tutores do IFRS - Campus Bento Gonçalves no mês de novembro de 2015. Este teve por objetivo avaliar o nível de aceitação de algumas das ferramentas desenvolvidas, obtendo uma taxa de resposta igual a $35 \%$.

De modo geral, o plugin de lembretes na página inicial do Moodle teve uma aceitação superior à dos relatórios de atividades. Em meio às características positivas avaliadas, destacaram-se os bons níveis de clareza e relevância das informações apresentadas (85,7\% e 78,6\%, respectivamente).

Observou-se, no entanto, que a maior parte dos respondentes não lembra de como era a página inicial do Moodle antes do plugin (64,3\%). Por esta razão, metade dos entrevistados não soube informar de que forma os lembretes auxiliam no gerenciamento de múltiplos cursos/tarefas ou mesmo se houve alguma melhoria neste aspecto. A falta de certeza para realizar tal avaliação pode ser resultado da pouca experiência com o ambiente virtual, bem como da falta de uso da ferramenta.

Ainda assim, a nova página inicial foi considerada muito atraente ou atraente por $85,7 \%$ dos professores-tutores. Os níveis de satisfação com a mesma variaram entre indiferente $(14,3 \%)$, satisfeito $(57,1 \%)$ e muito satisfeito $(28,6 \%)$ [Andrade et al. 2016].

Já nos relatórios de atividades (Resumo da Semana e Resumo do Dia), o índice de incerteza foi expressivo em diversas categorias, chegando a $30 \%$ em algumas delas. Isto demonstra que uma parte significativa dos entrevistados sequer percebeu a existência destas ferramentas.

Além disso, nem todos concordaram que receber as interações ocorridas no AVEA por e-mail é uma funcionalidade útil. Para estes, o volume de mensagens em sua caixa de entrada já é suficientemente grande e utilizar o relatório diário significaria aumentá-lo ainda mais. Desta forma, os níveis de satisfação para os relatórios de atividades variaram entre indiferente $(23,1 \%)$, satisfeito $(46,2 \%)$, muito satisfeito $(23,1 \%)$ e um usuário muito insatisfeito $(7,7 \%)$ [Andrade 2016 ].

\subsection{Análise de Registros do AVEA}

A análise das dimensões afetivas de professores-tutores do IFRS - Campus Bento Gonçalves buscou mensurar os aspectos de pontualidade, comprometimento, comunicabilidade, sociabilidade, iniciativa e meticulosidade dos docentes a partir dos registros de interação no ambiente virtual Moodle. Esta verificou bases de dados de dois momentos distintos no ano de 2015, tendo como referência as escalas e fórmulas propostas por Cunha (2009) no trabalho "Análise das Dimensões Afetivas do Tutor em Turmas de EaD".

A primeira base de dados corresponde ao final do primeiro semestre letivo (junho de 2015) e incluiu dados de 33 professores-tutores em 65 cursos. A segunda base de dados compreende o mês de novembro de 2015 e abrangeu dados de 60 professorestutores em 106 cursos.

De modo a verificar a influência positiva das ferramentas desenvolvidas na forma como professores-tutores utilizam o AVEA, os dados foram submetidos ao teste 
estatístico Teste $\mathrm{t}$ ou t-Student, o qual permite comparar amostras a uma população, duas amostras independentes ou ainda os valores de um mesmo sujeito em momentos distintos [Anderson et al. 2003]. Os cálculos estatísticos foram realizados através do software Microsoft Excel, sendo o nível de confiança aplicado igual a 95\%. Cada teste realizado verificou uma hipótese nula (onde os valores observados não seriam superiores na segunda amostra) e uma das hipóteses descritas na seção 2 (H1 a H6).

Para fins de comparação, a análise por professor-tutor considerou somente os docentes que utilizaram o Moodle em pelo menos um curso a cada semestre, de modo que houvesse valores para uma mesma dimensão afetiva em dois períodos distintos (amostras pareadas). O teste também foi aplicado ao conjunto de dados agrupado por curso. Neste caso, as amostras utilizadas presumiram variâncias diferentes.

A observância de uma dimensão afetiva está relacionada aos recursos do Moodle utilizados pelo professor-tutor durante um curso . Por esta razão, a quantidade de amostras é diferente para cada dimensão, variando entre 2 (pontualidade $\mathrm{e}$ comprometimento), 8 (sociabilidade), 9 (comunicabilidade) e 14 (iniciativa e meticulosidade) na análise por professor. Por outro lado, a análise por curso admitiu amostras de tamanhos diferentes para uma mesma dimensão. Assim, a quantidade mínima de observações nesta modalidade foi igual a 3 (nas dimensões pontualidade e comprometimento do primeiro semestre) e a máxima igual a 60 (na dimensão iniciativa do segundo semestre) [Andrade et al. 2016].

\subsection{Visão Geral}

Após analisadas todas as dimensões afetivas, percebeu-se que o uso de AVEAs na instituição ainda está aquém do esperado em diversos aspectos. A incorporação dos recursos já disponíveis no ambiente virtual, por exemplo, ainda não é uma unanimidade dentre os docentes, o que além de dificultar a mensuração de certas características, também põe à prova a relação entre as dificuldades enfrentadas por professores-tutores e a carência de ferramentas nele centradas.

Relacionando-se os registros do AVEA com o questionário de aceitação, pode-se perceber que a falta de familiaridade com o ambiente virtual também afeta a maneira como o usuário interage dentro do mesmo. Sendo a maior parte dos entrevistados usuários há menos de um ano, poucos reconheceram as mudanças do ambiente ou mesmo fizeram uso delas. De qualquer forma, puderam ser observados pequenos avanços entre o primeiro e segundo semestre em praticamente todas as dimensões. Ainda que o aumento de tais valores não configure uma regra geral, é possível que este decorra do conhecimento mais aprofundado do Moodle entre os dois períodos.

Dentre os usuários mais experientes, destacaram-se os excelentes níveis de comunicabilidade e iniciativa obtidos. A média de cursos por professor-tutor também foi mais alta entre estes usuários, o que demonstra que conseguem dedicar o tempo e atenção adequados a cada turma, mesmo com um número maior de tarefas e alunos para gerenciar. Ainda assim, outros aspectos como a meticulosidade estão longe do ideal, demonstrando que mesmo estes ainda tem pontos a melhorar.

O impacto das ferramentas desenvolvidas se deu justamente na meticulosidade, onde nenhum professor-tutor conseguiu manter uma média satisfatória. Os valores obtidos neste aspecto ainda podem e devem ser melhorados, mas já demonstram uma 
V Congresso Brasileiro de Informática na Educação (CBIE 2016)

Anais dos Workshops do V Congresso Brasileiro de Informática na Educação (CBIE 2016)

perspectiva de otimização a partir de ferramentas pensadas exclusivamente para apoiar o docente em suas tarefas diárias [Andrade et al. 2016].

\section{Conclusões}

Ao mesmo tempo em que demonstram potencial para enriquecer o processo ensino-aprendizagem, AVEAs representam um verdadeiro desafio para os professorestutores que precisam ou pretendem utilizá-los. A análise do primeiro questionário permitiu constatar que dificuldades são um aspecto intrínseco ao uso de ambientes virtuais, atingindo cerca de $90 \%$ dos 78 professores e pesquisadores entrevistados. O grupo incluiu desde usuários com menor conhecimento destes sistemas até aqueles que se dizem usuários avançados de AVEA.

$\mathrm{Na}$ maioria dos casos, percebeu-se que os problemas enfrentados pelo docente não estão restritos à falta de ferramentas adequadas no AVEA. Questões de infraestrutura, motivação, didática e capacitação tecnológica são fatores críticos para grande parte dos respondentes. Embora não estejam necessariamente relacionados à disponibilidade de recursos, acredita-se que estes tenham influência significativa na forma como os mesmos são assimilados.

As limitações decorrentes de uma carência de recursos ou funcionalidades no AVEA também tiveram grande destaque nas respostas ao questionário, tendo sido relatadas pela maioria dos participantes. Aspectos de usabilidade estão entre os requisitos mais mencionados, assim como relatórios de acompanhamento e assistentes inteligentes.

Por outro lado, percebeu-se que algumas das necessidades apontadas como "novas" já faziam parte do núcleo de ferramentas do AVEA, ou estavam disponíveis em extensões desenvolvidas por terceiros. A simples existência destas ferramentas não foi condição exclusiva para sua utilização. Assim, pode-se inferir que elas não são o único fator que distancia professores-tutores de um uso efetivo de AVEAs. Consequentemente, a hipótese principal "As dificuldades enfrentadas por professores e tutores no uso de AVEAs derivam da falta de ferramentas que ofereçam maior suporte à prática docente" pode ser considerada falsa.

Com base nos resultados expostos na Revisão Sistemática, constatou-se que a intenção de oferecer ferramentas voltadas a professores-tutores existe. A maior parte concentra-se em expandir as possibilidades tecnológicas, integrando o Moodle com softwares externos e exibindo dados de forma sumarizada. Nesta última, o professortutor é beneficiado de certa forma, pois não necessita percorrer várias páginas a fím de garantir que executou suas tarefas. Entretanto, ainda nota-se um distanciamento entre o que está disponível oficialmente e o que se pode oferecer através de ferramentas inteligentes.

Ao longo deste trabalho, foram desenvolvidos sete plugins para o ambiente Moodle. Os plugins desenvolvidos serviram ao propósito de responder a uma pergunta: "A partir do desenvolvimento de novas ferramentas, é possível promover um melhor uso do ambiente virtual por professores-tutores?". A este respeito, uma análise detalhada dos registros de interação no AVEA foi conduzida, abrangendo dados anteriores e posteriores ao uso das ferramentas. 
De acordo com os registros de interação do Moodle, a dimensão meticulosidade apresentou uma diferença positiva entre os dois períodos analisados. Acredita-se que este fato se deva à natureza dos plugins desenvolvidos, cujo foco foi justamente o de oferecer informações que permitissem acompanhar as interações realizadas no ambiente virtual. A partir das mesmas, o professor-tutor poderia decidir quando e onde sua atenção seria mais necessária. Assim, a hipótese secundária H6, "O uso das ferramentas propostas aumenta a meticulosidade do professor-tutor nos cursos oferecidos em AVEA" foi considerada verdadeira.

Por outro lado, não foi possível observar um aumento significativo nas dimensões pontualidade, comprometimento, comunicabilidade, sociabilidade e iniciativa. Portanto, as hipóteses secundárias $\mathrm{H} 1, \mathrm{H} 2, \mathrm{H} 3, \mathrm{H} 4$ e $\mathrm{H} 5$ foram consideradas falsas.

Vale ressaltar que as informações oferecidas nas novas ferramentas tiveram sua relevância reconhecida mesmo pelos usuários que alegaram não tê-las utilizado com propriedade. O questionário aplicado aos professores-tutores do IFRS - Campus Bento Gonçalves revelou certa falta de atenção às mudanças no AVEA, possivelmente motivada pela inexperiência com o ambiente. Ainda assim, o nível de satisfação com os plugins foi relativamente alto entre estes profissionais, especialmente no que diz respeito à ferramenta de lembretes na página inicial. A análise destas respostas indica que as ferramentas podem sim agregar valor à prática docente, desde que divulgadas e incorporadas ao dia-a-dia do professor-tutor.

De modo geral, conclui-se que as novas ferramentas apresentaram um impacto positivo na rotina de professores-tutores, auxiliando-os no acompanhamento de tarefas e gerenciamento de suas rotinas. Contudo, o ato de criá-las por si só não é suficiente para otimizar o processo educacional assistido por novas tecnologias. Para atingir tal objetivo, torna-se imprescindível que professores e tutores estejam cientes dos recursos disponíveis e, principalmente, que estes tomem a iniciativa de experimentá-los.

Por fim, as dificuldades de docentes em AVEAs não contempladas pelos plugins desenvolvidos estão sendo objeto de novos estudos. O projeto Minha Escola Virtual, por exemplo, foi concebido em meio a realização deste trabalho e consiste em um espaço sem fins lucrativos onde professores-tutores podem solicitar a criação de cursos no Moodle [Minha Escola Virtual, 2015].

Assim como os plugins de lembretes e relatórios, acredita-se que esta iniciativa contribuirá para a popularização dos recursos tecnológicos em salas de aula de todo o país, servindo também como ponto de partida para novas análises.

Como trabalhos futuros, pretende-se ampliar o alcance das ferramentas produzidas, disponibilizando-as para uso em outras instituições de ensino e por usuários interessados. A partir disto, será possível conduzir novas análises para verificar e, se possível, aprimorar o impacto positivo de tais extensões nas tarefas do professor-tutor [Andrade et al. 2016].

\section{Referências}

Almeida, M. (2003) "Educação a distância na internet: abordagens e contribuições dos ambientes digitais de aprendizagem". In: Educação e Pesquisa. v. 29 n. 2. FE/USP, São Paulo. 
V Congresso Brasileiro de Informática na Educação (CBIE 2016)

Anais dos Workshops do V Congresso Brasileiro de Informática na Educação (CBIE 2016)

Anderson, D., Sweeney, D., Williams, T. (2003) Estatística Aplicada à Administração e Economia, Pioneira Thomson Learning, $2^{\text {nd }}$ edition.

Andrade, F., Invernizzi, M., Rosito, M. and Silva, J. (2015) "Um aplicativo de lembrete de tarefas para alunos, pais e professores integrado ao Moodle". In: Anais do EATI Encontro Anual de Tecnologia da Informação e Semana Acadêmica de Tecnologia da Informação, ano 5 n. 1, Instituto Federal Farroupilha Campus Frederico Westphalen, Frederico Westphalen, p. 212-219.

Andrade, F. (2016) Análise de Ferramentas de Suporte às Tarefas do Professor-Tutor em um Ambiente Virtual de Ensino e Aprendizagem. Trabalho de Conclusão de Curso (Graduação em Tecnologia em Análise e Desenvolvimento de Sistemas). Instituto Federal de Educação, Ciência e Tecnologia do Rio Grande do Sul - Campus Bento Gonçalves, IFRS, Bento Gonçalves.

Cunha, C., Silva, J., Bercht, M. (2008) "Proposta de um modelo de atributos para o aprimoramento da comunicação afetiva para professores que atuam na educação a distância". In: Simpósio Brasileiro de Informática na Educação, Anais, SBIE, Fortaleza.

Cunha, F. (2009) Análise das dimensões afetivas do tutor em turmas de EaD. Trabalho de Conclusão de Curso (Graduação em Ciência da Computação). Universidade do Vale do Itajaí, UNIVALI, Itajaí.

Invernizzi, M., Petroli, F., Andrade, F., Rosito, M. and Silva, J. (2015) "Os Plugins Moodle para Automatização das Tarefas dos Docentes: uma revisão sistemática". In: XII ESUD - Congresso Brasileiro de Ensino Superior a Distância, Anais, UNEB, Salvador.

Kinshuk, Tretiakov, A., Hong, H. and Patel, A. (2001) "Human teacher in intelligent tutoring system: a forgotten entity". In: International Conference on Advanced Learning Technologies, Proceedings. IEEE, p. 227-230.

Mercado, L. (2002) Novas tecnologias na educação: reflexões sobre a prática, Edufal, Maceió.

Minha Escola Virtual, http://www.minhaescolavirtual.com.br, December 2015.

MOODLE (2015), http://www.moodle.org.br, December 2015.

Munera, D. (2015) "Themes: Archaius", https://moodle.org/plugins/theme_archaius, November 2015.

Silveira, D., Córdova, F. (2009) "A Pesquisa Científica", In: Métodos de Pesquisa , Organized by Tatiana Engel Gerhardt, Denise Tolfo Silveira. Editora UFRGS, Porto Alegre, p. 31-42.

Yacef, K. (2002) "Intelligent teaching assistant systems". In: International Conference on Computers in Education, Proceedings, IEEE, p. 136-140. 